\title{
MEFV mutation carriage in Israeli Jewish individuals from ethnicities with low risk for familial Mediterranean fever
}

\author{
Olga Feld ${ }^{1}$, Avi Livneh ${ }^{1}$, Yael Shinar ${ }^{2}$, Yaakov Berkun ${ }^{3}$ and Merav Lidar ${ }^{1}$
}

Familial Mediterranean fever (FMF) is a disease caused by mutations in the MEditerranean FeVer gene (MEFV), and in Israel it most commonly affects Jews of North African extraction, in whom the mutation carrier rate is as high as $\mathbf{1}$ in 5. To assess the protective as well as the modulating affect of MEFV mutation carriage on various inflammatory disease states, we sought to define the frequency of MEFV mutations in Israeli Jewish individuals of various ethnicities, including those with low frequency of FMF, which were not in the focus of our attention hitherto. A total of 163 adults of Bucharian, Turkish, Georgian, Yemenite and Bulgarian origin comprised the study group. The prevalence of the most frequent MEFV mutations in the Israeli Jewish population, namely: M694V, V726A and E148Q, was assessed. The association of mutation carriage with a personal history of FMF-like phenomena, as well as various inflammatory and non-inflammatory diseases, was evaluated. A high MEFV mutation frequency was found among Jews of Bucharian, Georgian and Bulgarian origin (20\%), whereas intermediate and low rates were detected in Jews of Turkish and Yemenite extraction (14 and $8 \%$, respectively). FMF-like manifestations and related diseases were observed more often in MEFV mutation carriers than in their counterparts. MEFV mutation frequency, directly assessed by DNA analysis, exceeds the rate calculated from disease prevalence in Israeli Jewish individuals originated from ethnicities with a low prevalence of FMF. MEFV mutation carriage in this subgroup is associated with various inflammatory disorders.

Journal of Human Genetics (2009) 54, 369-371; doi:10.1038/jhg.2009.33; published online 17 April 2009

Keywords: MEFV; FMF; ethnicity; Israeli-Jewish

\section{INTRODUCTION}

Familial Mediterranean fever (FMF) is an autoinflammatory, autosomal recessive disorder, prevalent in the Mediterranean basin and characterized by recurrent attacks of fever and serositis. The MEditerranean FeVer gene (MEFV) encodes a protein, called pyrin/marenostrin, which is involved in the regulation of inflammation and apoptosis. ${ }^{1}$ Mutations in MEFV (more than 50 thus far) have been associated with FMF. In Israel, FMF most commonly affects Jews of North African and Iraqi extraction (Sepharadi Jews), in whom disease frequency is 1:200 and 1:1000, respectively, ${ }^{2}$ whereas in Ashkenazi Jews, originating from Central Europe, the disorder is rare with a frequency of 1:160 000 only. ${ }^{3}$ However, direct assessment of mutation frequency, as reported in several studies, revealed quite surprising results of extremely higher-than-expected rates. ${ }^{4-6}$ Furthermore, the mutation rates in these two ethnic groups, so vastly separated by FMF disease frequency, were quite comparable, 1 in 5 vs 1 in $7-8$ in Sepharadi and Ashkenazi Jews, respectively. These findings suggest that only direct testing of MEFV mutation frequency is appropriate for the assessment of its role.
This study aims to define the frequency of MEFV mutations in the Israeli-Jewish population with low risk for FMF, specifically those originated from the Jewish diaspora of Buchara, Georgia, Yemen, Turkey and Bulgaria. Knowledge of the prevalence of MEFV mutation carriage may help in elucidating their biological advantage and their role in the modulation of non-FMF inflammatory disorders.

\section{MATERIALS AND METHODS}

The study group included 163 Jewish individuals, born to parents, both originating from the same country, Buchara, Turkey, Georgia, Yemen or Bulgaria. They were enrolled, provided they stated no personal or family history of FMF. Participants were interviewed, and a questionnaire focusing on manifestations indicative of inflammatory diseases was completed. The study was approved by the institutional review board, and all participants signed an informed consent.

DNA was analyzed for the three most common Jewish MEFV mutations (M694V, V726A, E148Q), using PCR amplification and restriction enzyme digestion. $^{7}$

Statistical analysis was performed for categorical variables using $\chi^{2}$-test or Fisher's exact test, according to the size of the cells examined; and for

${ }^{1}$ Department of Medicine F, Sheba Medical Center, Heller Institute of Medical Research, Ramat Gan, Israel; ${ }^{2}$ Sheba Medical Center, Heller Institute of Medical Research, Ramat Gan, Israel and ${ }^{3}$ Department of Pediatrics, Sheba Medical Center, Sackler School of Medicine, Heller Institute of Medical Research, Tel Aviv University, Tel Aviv, Israel Correspondence: Dr M Lidar, Department of Medicine F, Sheba Medical Center, Heller Institute of Medical Research, Tel-Hashomer, Ramat Gan 52621, Israel. E-mail: merav.lidar@sheba.health.gov.il

Received 14 December 2008; revised 1 March 2009; accepted 16 March 2009; published online 17 April 2009 
continuous variables using the Student's $t$-test. All tests of significance were two-tailed; $P$-values $<0.05$ were considered statistically significant.

\section{RESULTS}

A total of 163 individuals, originating from ethnicities with low rates of FMF, were tested for mutations in the MEFV gene. Baseline demographic data are summarized in Table 1 . There were 27 carriers of MEFV mutations, 2 of whom, with no manifestations of FMF, carried two mutations each. The mean age and gender distribution were equal among carriers and non-carriers of an MEFV mutation (58.3 \pm 17 vs $61.9 \pm 19$ years and male/female ratio of $16 / 11$ and $72 / 64$, respectively). Mutation carriage rate and distribution of the three studied mutations by origin are detailed in Table 2. The E148Q mutation was the most prevalent, found in 19/326 chromosomes (6\%).

The rate of FMF-like conditions, which may indicate a hitherto unrecognized FMF, amyloidosis, splenomegaly (indicative of subclinical inflammation) and a long list of inflammatory diseases, more commonly encountered in FMF, among mutation carriers and non-carriers, are listed in Table 3. There was a statistically significant excess of the combined frequency of FMF-like manifestations (acute arthritis, pericarditis, erysipelas episodes and Behçet's-like disease) in MEFV mutation carriers ( $P=0.001$ for the combination). No difference was noted in the frequency of non-inflammatory diseases aside from hypertension, which was unexplainably overrepresented among non-carriers $(67 / 136$ vs $7 / 27$ in carriers, $P=0.03$, respectively).

\section{DISCUSSION}

The rate of MEFV mutation carriage in Israeli Jewish individuals from ethnicities with a low risk for FMF may be as high as $20 \%$, reaching the frequencies described in North-African Jews, in which overt FMF

Table 1 Demographic characteristics of study participants by origin

\begin{tabular}{lclc}
\hline Country of origin & Number of individuals & M/F & Age (years \pm s.d.) \\
\hline Buchara & 38 & $15 / 23$ & $50.5 \pm 19.4$ \\
Georgia & 41 & $22 / 19$ & $64.7 \pm 16.0$ \\
Yemen & 36 & $18 / 18$ & $62.6 \pm 22.6$ \\
Turkey & 29 & $23 / 6$ & $63.5 \pm 14.7$ \\
Bulgaria & 19 & $10 / 9$ & $67.7 \pm 15.2$ \\
Total & 163 & $88 / 75$ & $61.1 \pm 18.9$ \\
\hline
\end{tabular}

Abbreviations: $F$, female; $M$, male. is prevalent. The combination, in these ethnicities, of a high mutation frequency, on the one hand, with a low disease prevalence, on the other, indicates low mutation penetrance. Indeed, E148Q and V726A, which are considered 'weak' mutations, ${ }^{8}$ were the most commonly found in most ethnic groups except for Georgian individuals, in whom M694V, which is associated with a more severe disease phenotype, as well as amyloidosis, ${ }^{9}$ was commonly detected (M694V/E148Q ratio of 4/5).

The finding of a high mutation frequency in Jewish communities immigrated to Israel from Eastern Europe, supports the view that this population has actually emerged originally from an area endemic to a hitherto unrecognized pathogen, from which the mutations protect, comparable with Jewish subgroups with high risk for FMF.

On the basis of our experience at the National Center for FMF, which holds in its registry data on most FMF patients in Israel, the frequency of FMF in Georgian Jews is very low (1:5000). This suggests that Georgian Jews carry a modifier gene or system, or are exposed to an environmental moderating factor, which inhibits the clinical expression of the most penetrant MEFV mutation, M694V.

Finally, our findings reiterate the higher frequency of phenotype 3 (genetic positive, clinically negative individuals) in Jewish individuals and the occurrence of FMF-like symptoms and inflammatory diseases in MEFV mutation carriers. The reduced rate of hypertension in mutation carriers needs to be ascertained and further investigated.

Table 3 Frequency of FMF-related conditions

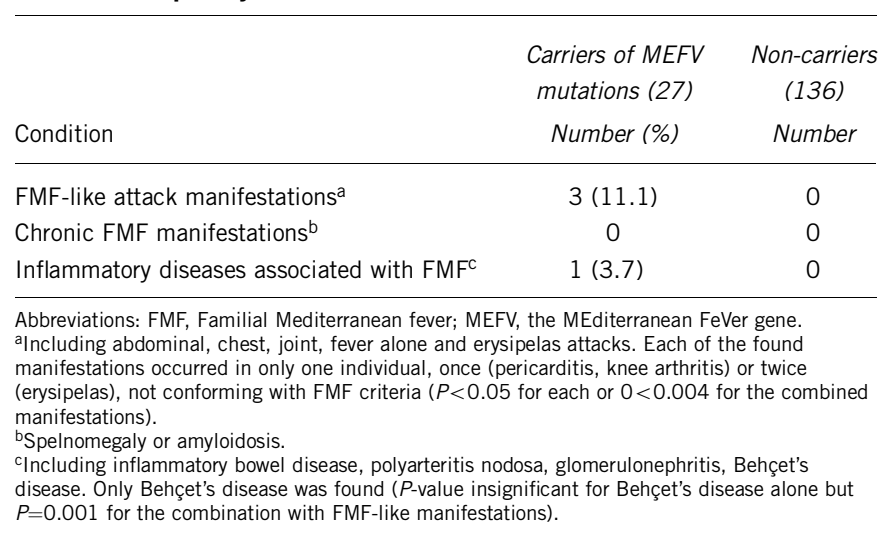

Table 2 MEFV mutation by ethnic origin

\begin{tabular}{|c|c|c|c|c|c|c|c|c|}
\hline \multirow[b]{2}{*}{ Country of origin } & \multirow[b]{2}{*}{ Number of subjects } & \multicolumn{3}{|c|}{ Number of mutations } & \multirow[b]{2}{*}{ Carrier rate } & \multicolumn{3}{|c|}{ Mutation type } \\
\hline & & 0 & 1 & 2 & & M694V & $V 726 A$ & $E 148 Q$ \\
\hline Buchara & 38 & 30 & 8 & 0 & $(1: 5) 0.2$ & 0 & 0 & 8 \\
\hline Georgia & 41 & 33 & 7 & 1 & $(1: 5) 0.2$ & 4 & 0 & 5 \\
\hline Yemen & 36 & 33 & 3 & 0 & $(1: 12-13) 0.08$ & 1 & 0 & 2 \\
\hline Turkey & 29 & 25 & 4 & 0 & $(1: 7-8) 0.14$ & 1 & 2 & 1 \\
\hline Bulgaria & 19 & 15 & 3 & 1 & $(1: 5) 0.2$ & 0 & 2 & 3 \\
\hline Total & 163 & 136 & 25 & 2 & $(1: 5-6) 0.18$ & 6 & 4 & 19 \\
\hline
\end{tabular}

Abbreviations: MEFV, the MEditerranean FeVer gene. 
1 Livneh, A., Drenth, J. P., Klasen, I. S., Langevitz, P., George, J., Shelton, D. A. et al. Familial Mediterranean fever and hyperimmunoglobulinemia D syndrome: two diseases with distinct clinical, serologic, and genetic features. J. Rheumatol. 24, 1558-1563 (1997).

2 Pras, E., Livneh, A., Balow, J. E. Jr, Pras, E., Kastner, D. L. Pras, M. et al Clinical differences between North African and Iraqi Jews with familial Mediterranean fever. Am. J. Med. Genet. 75, 216-219 (1998).

3 Heller, H., Sohar, E. \& Pras, M. Ethnic distribution and amyloidosis in familial Mediterranean fever (FMF). Pathol. Microbiol. (Basel) 24, 718-723 (1961).

4 Stoffman, N., Magal, N., Shohat, T., Lotan, R., Koman, S., Oron, A. et al. Higher than expected carrier rates for familial Mediterranean fever in various Jewish ethnic groups. Eur. J. Hum. Genet. 8, 307-310 (2000).

5 Shinawi, M., Brik, R., Berant, M., Kasinetz, L. \& Gershoni-Baruch, R. Familial Mediterranean fever: high gene frequency and heterogeneous disease among an Israeli-Arab population. J. Rheumatol. 27, 1492-1495 (2000).
6 Kogan, A., Shinar, Y., Lidar, M., Revivo, A., Langevitz, P., Padeh, S. et al. Common MEFV mutations among Jewish ethnic groups in Israel: high frequency of carrier and phenotype III states and absence of a perceptible biological advantage for the carrier state. Am. J. Med. Genet. 102, 272-276 (2001).

7 Livneh, A., Langevitz, P., Shinar, Y., Zaks, N., Kastner, D. L., Pras, M. et al. MEFV mutation analysis in patients suffering from amyloidosis of familial Mediterranean fever. Amyloid 6, 1-6 (1999).

8 Shinar, Y., Livneh, A., Langevitz, P., Zaks, N., Aksentijevich, I., Koziol, D. E. et al. Genotype-phenotype assessment of common genotypes among patients with familial Mediterranean fever. J. Rheumatol. 27, 1703-1707 (2000).

9 Gershoni-Baruch, R., Brik, R., Zacks, N., Shinawi, M., Lidar, M. \& Livneh, A. The contribution of genotypes at the MEFV and SAA1 loci to amyloidosis and disease severity in patients with familial Mediterranean fever. Arthritis Rheum. 48, 1149-1155 (2003). 